\title{
Study on subclasses of analytic functions
}

\author{
Imran Faisal \\ Department of Mathematics, \\ University of Education, Pakistan \\ email: faisalmath@gmail.com
}

\author{
Maslina Darus \\ School of Mathematical Sciences, \\ Faculty of Science and Technology, \\ Universiti Kebangsaan Malaysia, \\ Malaysia \\ email: maslina@ukm.edu.my
}

\begin{abstract}
By making use of new linear fractional differential operator, we introduce and study certain subclasses of analytic functions associated with Symmetric Conjugate Points and defined in the open unit disk $\mathbb{U}=\{z:|z|<1\}$. Inclusion relationships are established and convolution properties of functions in these subclasses are discussed.
\end{abstract}

\section{Introduction and preliminaries}

Let $A$ denote the class of functions of the form

$$
f(z)=z+\sum_{k=2}^{\infty} a_{k} z^{k},
$$

which are analytic in the open unit disk $\mathbb{U}=\{z:|z|<1\}$ and normalized by $f(0)=f^{\prime}(0)-1=0$.

A function $f \in A$ is called starlike if and only if

$$
\mathfrak{R}\left(\frac{z f^{\prime}(z)}{f(z)}\right) \geq 0,(z \in \mathbb{U}) .
$$

2010 Mathematics Subject Classification: 30C45

Key words and phrases: starlike functions, symmetric conjugate points, inclusion relationships 
The class of starlike functions is denoted by $S$.

A function $f \in A$ is called convex if and only if

$$
\mathfrak{R}\left(1+\frac{z f^{\prime \prime}(z)}{f^{\prime}(z)}\right) \geq 0,(z \in \mathbb{U}) .
$$

The class of convex functions is denoted by $\mathrm{K}$.

A function $f \in A$ is called starlike of order $\rho$ if and only if

$$
\mathfrak{R}\left(\frac{z f^{\prime}(z)}{f(z)}\right) \geq \rho,(\rho>0, z \in \mathbb{U}) .
$$

The class of starlike functions of order $\rho$ is denoted by $S V^{\star}(\rho)$.

Similarly a function $f \in A$ is called convex of order $\rho$ if and only if

$$
\mathfrak{R}\left(1+\frac{z f^{\prime \prime}(z)}{f^{\prime}(z)}\right) \geq \rho,(\rho>0, z \in \mathbb{U}) .
$$

The class of starlike functions of order $\rho$ is denoted by $K V(\rho)$.

It follows from (2) and (3) that $f \in K V(\rho)$ if and only if $z f^{\prime}(z) \in S V^{\star}(\rho)$.

Let $f \in A$ and $g \in S V^{\star}(\rho)$, then $f \in A$ is called close-to-convex of order $\theta$ and type $\rho$ if and only if

$$
\mathfrak{R}\left(\frac{z f^{\prime}(z)}{g(z)}\right) \geq \theta,(0 \leq \theta, \rho<1, z \in \mathbb{U}) .
$$

The class of close-to-convex of order $\theta$ and type $\rho$ is denoted by $\operatorname{CV}(\theta, \rho)$.

In 1959, Sakaguchi [1] introduced the following class of analytic functions:

A function $f \in A$ is called starlike with respect to symmetrical points, and its class is denoted by $\mathrm{SV}_{s}$, if it satisfies the analytic criterion

$$
\mathfrak{R}\left(\frac{z f^{\prime}(z)}{f(z)-f(-z)}\right)>0,(z \in \mathbb{U}) .
$$

For more details we refer to study Shanmugam et al. [2], Chand and Singh [3] and Das and Singh [4] respectively.

In 1987, El-Ashwah and Thomas [5] introduced the following class of analytic functions:

A function $f \in A$ is called starlike with respect to symmetric conjugate points, and its class is denoted by $\mathrm{SV}_{\mathrm{sc}}$, if it satisfies the analytic criterion

$$
\mathfrak{R}\left(\frac{z f^{\prime}(z)}{f(z)-\overline{f(-z)}}\right)>0,(z \in \mathbb{U}) .
$$


For two functions $f$ and $g$ analytic in $\mathbb{U}$, we say that the function $f$ is subordinate to $g$ in $\mathbb{U}$ and write

$$
\mathrm{f}(z) \prec \mathrm{g}(z)(z \in \mathbb{U}),
$$

if there exists a Schwarz function $w(z)$ which (by definition) is analytic in $\mathbb{U}$ with

$$
w(0)=0 \text { and }|w(z)|<1
$$

such that

$$
f(z)=g(w(z)) z \in \mathbb{U} .
$$

Indeed it is known that

$$
\mathrm{f}(z) \prec \mathrm{g}(z) z \in \mathbb{U} \Rightarrow \mathrm{f}(0)=\mathrm{g}(0) \text { and } \mathrm{f}(\mathbb{U}) \subset \mathrm{g}(\mathbb{U}) .
$$

Furthermore, if the function $g$ is univalent in $\mathbb{U}$, then we have the following equivalence:

$$
\mathrm{f}(z) \prec \mathrm{g}(z) z \in \mathbb{U} \Leftrightarrow \mathrm{f}(0)=\mathrm{g}(0) \text { and } \mathrm{f}(\mathbb{U}) \subset \mathrm{g}(\mathbb{U}) .
$$

For functions $f(z)=z+\sum_{k=2}^{\infty} a_{k} z^{k}$ and $g(z)=z+\sum_{k=2}^{\infty} b_{k} z^{k}$ the Hadamard product (or convolution) $f * g$ is defined as usual by

$$
(f * g)(z)=z+\sum_{k=2}^{\infty} a_{k} b_{k} z^{k}
$$

Let

$$
\varphi(\mathrm{a}, \mathrm{c} ; z)=z_{2} \mathrm{~F}(1, \mathrm{a} ; \mathrm{c} ; z)=\sum_{\mathrm{k}=0}^{\infty} \frac{(\mathrm{a})_{\mathrm{k}}}{(\mathrm{c})_{\mathrm{k}}} z^{\mathrm{k}-1},(z \in \mathbb{U} ; \mathrm{c} \neq 0,-1,-2, . \mathrm{s}),
$$

where $(a)_{k}$ is the pochhammer symbol defined by

$$
(a)_{k}=\frac{\Gamma(k+a)}{\Gamma(a)}=\left\{\begin{array}{ll}
1 & \text { if } k=0 \\
a(a+1)(a+2) \cdot s(a+k-1) & \text { if } k \in N
\end{array}\right\} .
$$

In 1987, Owa and Srivastava [7] introduced the operator as follow

$$
\begin{aligned}
\Omega^{\alpha} \mathrm{f}(z) & =\Gamma(2-\alpha) z^{\alpha} \mathrm{D}_{z}^{\alpha} \mathrm{f}(z)=\varphi(2,2-\alpha ; z) * \mathrm{f}(z), \\
\alpha & \neq 2,3,4, \ldots
\end{aligned}
$$


Also note that $\Omega^{0} f(z)=f(z)$ and $D_{z}^{\alpha} f(z)$ is the fractional derivative of order $\alpha$ given in [6].

For $f \in A$, we define the linear fractional differential operator as follow:

$$
\begin{aligned}
I_{\lambda}^{0, v}(\alpha, \beta, \mu) f(z) & =f(z) \\
I_{\lambda}^{1, v}(\alpha, \beta, \mu) f(z) & =\left(\frac{v-\mu+\beta-\lambda}{v+\beta}\right) \Omega^{\alpha} f(z)+\left(\frac{\mu+\lambda}{v+\beta}\right) z\left(\Omega^{\alpha} f(z)\right)^{\prime} \\
I_{\lambda}^{2, v}(\alpha, \beta, \mu) f(z) & =I_{\lambda}^{\alpha}\left(I_{\lambda}^{1, v}(\alpha, \beta, \mu) f(z)\right) \\
& \vdots \\
I_{\lambda}^{n, v}(\alpha, \beta, \mu) f(z) & =I_{\lambda}^{\alpha}\left(I_{\lambda}^{n-1, v}(\alpha, \beta, \mu) f(z)\right) .
\end{aligned}
$$

If $f(z)$ is given by (1) then from (5) we have

$$
\begin{aligned}
& \mathrm{I}_{\lambda}^{\mathrm{n}, \nu}(\alpha, \beta, \mu) f(z) \\
& =z+\sum_{\mathrm{k}=2}^{\infty}\left(\left(\frac{\Gamma(\mathrm{k}+1) \Gamma(2-\alpha)}{\Gamma(k+1-\alpha)}\right)\left(\frac{v+(\mu+\lambda)(\mathrm{k}-1)+\beta}{v+\beta}\right)\right)^{n} \mathrm{a}_{\mathrm{k}} z^{\mathrm{k}} .
\end{aligned}
$$

Using (4) we conclude that

$$
\mathrm{I}_{\lambda}^{\mathrm{n}, v}(\alpha, \beta, \mu) \mathrm{f}(z)=\underbrace{\left[\varphi(2,2-\alpha ; z) * \mathrm{~g}_{\beta, \lambda}^{\mu, \nu}(z) . s \varphi(2,2-\alpha ; z) * \mathrm{~g}_{\beta, \lambda}^{\mu, v}(z)\right]} * \mathrm{f}(z),
$$

where

$$
\begin{aligned}
g_{\beta, \lambda}^{\mu, v}(z) & =\frac{z-\left(\frac{v-\mu+\beta-\lambda}{v+\beta}\right) z^{2}}{(1-z)^{2}} \\
& =\left(z-\frac{v-\mu+\beta-\lambda}{v+\beta} z^{2}\right)\left(1+2 z+3 z^{2}+\cdots\right) \\
& =z+\left(1+\frac{\mu+\lambda}{v+\beta}\right) z^{2}+\left(1+2 \frac{\mu+\lambda}{v+\beta}\right) z^{3}+\cdots \\
& \vdots \\
g_{\beta, \lambda}^{\mu, v}(z) & =z+\sum_{k=2}^{\infty}\left(\frac{v+(\mu+\lambda)(k-1)+\beta}{v+\beta}\right) z^{k} .
\end{aligned}
$$

$$
\varphi(2,2-\alpha ; z) * g_{\beta, \lambda}^{\mu, v}(z) . s \varphi(2,2-\alpha ; z) * g_{\beta, \lambda}^{\mu, v}(z)=\text { n-times product. }
$$


Similarly, we find the following:

$$
\begin{aligned}
g_{\beta, \lambda}^{\mu}(z) & =z+\sum_{k=2}^{\infty}\left(\frac{\beta+(\mu+\lambda)(k-1)}{\beta}\right) z^{k} . \\
g_{\beta, \lambda}^{v}(z) & =z+\sum_{k=2}^{\infty}\left(\frac{v+\lambda(k-1)+\beta}{v+\beta}\right) z^{k} . \\
g_{\beta}^{v}(z) & =z+\sum_{k=2}^{\infty}\left(\frac{v+(k-1)+\beta}{v+\beta}\right) z^{k} . \\
g_{\lambda}^{\mu}(z) & =z+\sum_{k=2}^{\infty}(1+(\mu+\lambda)(k-1)) z^{k} . \\
g_{\lambda}(z) & =z+\sum_{k=2}^{\infty}(1+\lambda(k-1)) z^{k} .
\end{aligned}
$$

Further, a straightforward calculation reveals that many differential operators introduced in other papers are special cases of the differential operator defined by (6) which generalizes some well known differential operators.

1. $\beta=1, \mu=0, \alpha=0$, we obtain, Aouf et al. differential operator [8].

2. $\alpha=0$, we obtain differential operator of Darus and Faisal [29].

3. $\beta=0, \alpha=0$, we obtain differential operator Darus and Faisal [30].

4. $v=1, \beta=0, \mu=0, \alpha=0$, we obtain, Al-Oboudi differential operator [9].

5. $v=1, \beta=0, \mu=0, \lambda=1, \alpha=0$, we obtain, Sălăgean's operator [10].

6. $\beta=l, \mu=0, \alpha=p$, we obtain, A. Catas operator [26].

7. $\nu=1, \beta=0, \mu=0$, we obtain, Al-Oboudi-Al-Amoudi operator [14, 25].

8. $\beta=1, \lambda=1, \mu=0, \alpha=0$, we obtain, Cho-Srivastava operator $[12,13]$.

9. $\nu=1, \beta=1, \lambda=1, \mu=0, \alpha=0$, we obtain, Uralegaddi-Somanatha operator [11].

10. $v=1, \beta=0, \mu=0, \lambda=0, n=1$, we obtain, Owa-Srivastava operator [7]. 
11. $\beta=l, \mu=0, \alpha=p, \lambda=1$, we obtain, Kumar et al. and Srivastava et al. operators $[27,28]$.

For $f \in A$, we define $f_{m}$ by

$$
\left.f_{m}(z)=\frac{1}{2 m} \sum_{k=0}^{m-1}\left[\omega^{-k} f\left(\omega^{k} z\right)+\omega^{k} \overline{f\left(\omega^{k} \bar{z}\right.}\right)\right],
$$

where $m$ be a positive integer and $\omega=\exp (2 \pi /(m))$.

A function $f \in A$ is called $\lambda$-starlike with respect to $2 m$-symmetric conjugate points and its class is denoted by $\operatorname{SV}_{m}(\lambda)$, if it satisfy the analytic criterion

$$
\mathfrak{R}\left(\frac{(1-\lambda) z f^{\prime}(z)+\lambda\left(z f^{\prime}(z)\right)^{\prime}}{(1-\lambda) f_{m}(z)+\lambda z f_{m}^{\prime}(z)}\right)>0,(z \in U, \lambda \geq 0),
$$

where $f_{m}$ is given by (14). For details about $S V_{m}(\lambda)$, we refer to study $[15,16$, 17, 18].

By using (14), we have

$$
\begin{aligned}
& \left.f_{m}(z)=\frac{1}{2 m} \sum_{k=0}^{m-1}\left\{\omega^{-k} f\left(\omega^{k} z\right)+\omega^{k} \overline{f\left(\omega^{k} \bar{z}\right.}\right)\right\} \text {, implies } \\
& \mathrm{I}_{\lambda}^{\mathrm{n}, v}(\alpha, \beta, \mu) \mathrm{f}_{\mathrm{m}}(z)=\frac{1}{2 \mathrm{~m}} \sum_{\mathrm{k}=0}^{\mathrm{m}-1}\left\{\omega^{-\mathrm{k}} \mathrm{I}_{\lambda}^{\mathrm{n}, v}(\alpha, \beta, \mu) \mathrm{f}\left(\omega^{\mathrm{k}} z\right)\right. \\
& \left.\left.+\omega^{k} \overline{I_{\lambda}^{n, v}(\alpha, \beta, \mu) f\left(\omega^{k} \bar{z}\right.}\right)\right\}, \\
& z\left(I_{\lambda}^{n, v}(\alpha, \beta, \mu) f_{m}(z)\right)^{\prime}=\frac{1}{2 m} \sum_{k=0}^{m-1}\left\{\omega^{-k} z\left(I_{\lambda}^{n, v}(\alpha, \beta, \mu) f\left(\omega^{k} z\right)\right)^{\prime}\right. \\
& \left.\left.+\omega^{k} z\left(\overline{D_{\lambda}^{n, v}(\alpha, \beta, \mu) f\left(\omega^{k} \bar{z}\right.}\right)\right)^{\prime}\right\}, \\
& I_{\lambda}^{n, v}(\alpha, \beta, \mu)\left(z f_{m}^{\prime}(z)\right)=\frac{1}{2 m} \sum_{k=0}^{m-1}\left\{I_{\lambda}^{n, v}(\alpha, \beta, \mu)\left(z f^{\prime}\left(\omega^{k} z\right)\right)\right. \\
& \left.+\overline{\mathrm{I}_{\lambda}^{n, v}(\alpha, \beta, \mu)\left(z f^{\prime}\left(\omega^{k} \bar{z}\right)\right.}\right\}, \\
& I_{\lambda}^{n, v}(\alpha, \beta, \mu) f_{m}\left(\omega^{j} z\right)=\omega^{j} I_{\lambda}^{n, v}(\alpha, \beta, \mu) f_{m}(z) \text {, } \\
& \mathrm{I}_{\lambda}^{\mathrm{n}, v}(\alpha, \beta, \mu) \mathrm{f}_{\mathrm{m}}(\bar{z})=\overline{\mathrm{I}_{\lambda}^{\mathrm{n}, v}(\alpha, \beta, \mu) \mathrm{f}_{\mathrm{m}}(z)} .
\end{aligned}
$$

Next we introduce new subclasses of analytic functions in $\mathbb{U}$ associated with linear fractional differential operator $\operatorname{In}_{\lambda}^{\mathfrak{n}, v}(\alpha, \beta, \mu) f(z)$, as follow; 
Definition 1 For $n \in \mathbb{N} \cup\{0\}, m \in \mathbb{N}$ and $\alpha, \beta, \lambda, \mu, v \geq 0$, let $\mathcal{S} \mathcal{V}_{m, \lambda}^{\mathrm{n}, v}(\alpha, \beta, \mu)(\mathrm{h})$ denote the class of functions $f$ defined by (1) and satisfying the analytic criterion

$$
\frac{z\left(I_{\lambda}^{n, v}(\alpha, \beta, \mu) f(z)\right)^{\prime}}{I_{\lambda}^{n, v}(\alpha, \beta, \mu) f_{m}(z)} \prec h(z), \quad z \in \mathbb{U}
$$

where $\mathrm{h}$ is a convex function in $\mathbb{U}$ with $\mathrm{h}(0)=1$.

Definition 2 Let $\mathcal{K} \mathcal{V}_{m, \lambda}^{n, v}(\alpha, \beta, \mu)(h)$ denote the class of functions $\mathrm{f}$ defined by (1) and satisfying the analytic criterion if $\frac{\mathrm{I}_{\lambda}^{\mathrm{n}, \nu}(\alpha, \beta, \mu) g_{\mathrm{m}}(z)}{z} \neq 0$ and

$$
\frac{z\left(I_{\lambda}^{n, v}(\alpha, \beta, \mu) f(z)\right)^{\prime}}{I_{\lambda}^{n, v}(\alpha, \beta, \mu) g_{m}(z)} \prec h(z), \quad z \in \mathbb{U},
$$

for some $\mathrm{g} \in \mathcal{S} \mathcal{V}_{\mathrm{m}, \lambda}^{\mathrm{n}, \boldsymbol{v}}(\alpha, \beta, \mu)(\mathrm{h})$.

Remark 1 In 2010, F. M. Al-oboudi [25], introduced certain subclasses of analytic functions which contains only functions of the form given in (13), but there were infinite analytic functions in the open unit disk $\mathbb{U}$, of the form given in (8), (9), (10), (11) and (12) respectively, which were out of range of the classes given in [25]. Therefore it was necessary to find out or to introduce a new differential operator of the form (6). We introduce the subclasses $\mathcal{S} \mathcal{V}_{m, \lambda}^{n, v}(\alpha, \beta, \mu)(h)$ and $\mathcal{K} \mathcal{V}_{m, \lambda}^{n, v}(\alpha, \beta, \mu)(h)$ of analytic functions by using such differential operator, with a different approach that includes all functions of the form given in (8) to (13).

\section{Main results}

In this section, we have discussed inclusion relations as well as convolution properties for the function belonging to the classes $\mathcal{S} \mathcal{V}_{m, \lambda}^{\mathrm{n}, \boldsymbol{v}}(\alpha, \beta, \mu)(\mathrm{h})$ and $\mathcal{K} \mathcal{V}_{m, \lambda}^{n, v}(\alpha, \beta, \mu)(h)$ respectively.

Lemma 1 [19] Let $\mathbf{f}$ and $\mathbf{g}$ be starlike functions of order $1 / 2$ then so is $\mathbf{f} * \mathbf{g}$.

Lemma 2 [20] Let $\mathrm{P}$ be a complex function in $\mathbb{U}$ with $\mathfrak{R}(\mathrm{P}(z))>0$ for $z \in \mathbb{U}$ and let $\mathrm{h}$ be a convex function in $\mathbb{U}$. If $\mathrm{p}$ is analytic in $\mathbb{U}$ with $\mathrm{p}(0)=\mathrm{h}(0)$ and if

$$
p(z)+P(z) z p^{\prime}(z) \prec h(z)
$$

then $\mathrm{p}(z) \prec \mathrm{h}(z)$. 
Lemma 3 [21] Let $\mathrm{c}>-1$ and let $\mathrm{I}_{\mathrm{c}}: \mathrm{A} \rightarrow \mathrm{A}$ be the integral operator defined by $\mathrm{F}=\mathrm{I}_{\mathrm{c}}(\mathrm{f})$, where

$$
F(z)=\frac{c+1}{z^{c}} \int_{0}^{z} t^{c-1} f(t) d t .
$$

Let $\mathrm{h}$ be a convex function, with $\mathrm{h}(0)=1$ and then $\mathfrak{R}(\mathrm{h}(z)+\mathrm{c})>0, z \in \mathbb{U}$. If $\mathrm{f} \in \mathrm{A}$ and $\frac{z \mathrm{f}^{\prime}(z)}{\mathrm{f}(z)} \prec \mathrm{h}(z)$, then

$$
\frac{z \mathrm{~F}^{\prime}(z)}{\mathrm{F}(z)} \prec \mathrm{q}(z) \prec \mathrm{h}(z),
$$

where $\mathrm{q}$ is univalent and satisfies the differential equation

$$
\mathrm{q}(z)+\frac{z \mathrm{q}^{\prime}(z)}{\mathrm{q}(z)+\mathrm{c}}=\mathrm{h}(z) .
$$

Lemma 4 [22] Let $\mathbf{f}$ and $\mathrm{g}$, respectively be in the classes $\mathrm{K}$ and $\mathrm{S}$, then for every function $\mathrm{F} \in \mathrm{A}$, we have

$$
\frac{(\mathrm{f}(z) * \mathrm{~g}(z) \mathrm{F}(z))}{(\mathrm{f}(z) * \mathrm{~g}(z))} \in \overline{\mathrm{co}}(\mathrm{F}(\mathbb{U})), \quad z \in \mathbb{U}
$$

where $\overline{\mathrm{co}}$ denotes the closed convex hull.

Lemma 5 [22] Let $\mathrm{f}$ and $\mathrm{g}$ be univalent starlike of order $\frac{1}{2}$ for every function $\mathrm{F} \in \mathrm{A}$, we have

$$
\frac{(\mathrm{f}(z) * \mathrm{~g}(z) \mathrm{F}(z))}{(\mathrm{f}(z) * \mathrm{~g}(z))} \in \overline{\mathrm{co}}(\mathrm{F}(\mathbb{U})), \quad z \in \mathbb{U}
$$

where $\overline{\mathrm{co}}$ denotes the closed convex hull.

Theorem 1 Let $\mathrm{h}$ be a convex function in $\mathbb{U}$ with $\mathrm{h}(0)=1, \overline{\mathrm{h}(\bar{z})}=\mathrm{h}(\mathrm{z})$ and let $\mu+\lambda \geq v+\beta$, if $f \in \mathcal{S V}_{m, \lambda}^{\mathrm{n}, \nu}(\alpha, \beta, \mu)(\mathrm{h})$ then

$$
\frac{z\left(I_{\lambda}^{n, v}(\alpha, \beta, \mu) f_{m}(z)\right)^{\prime}}{I_{\lambda}^{n, v}(\alpha, \beta, \mu) f_{m}(z)} \prec h(z), z \in \mathbb{U} .
$$

Moreover, if $\mathfrak{R}\left(h(z)+\frac{v+\beta-\mu-\lambda}{\mu+\lambda}\right)>0$ in $\mathbb{U}$ then

$$
\frac{z\left(I _ { \lambda } ^ { n - 1 , v } \left(\alpha, \beta, \mu\left(\left(\Omega^{\alpha} f_{m}(z)\right)\right)^{\prime}\right.\right.}{I_{\lambda}^{n-1, v}(\alpha, \beta, \mu)\left(\Omega^{\alpha} f_{m}(z)\right)} \prec q(z) \prec h(z), z \in \mathbb{U} .
$$


Where $\mathrm{q}$ is the univalent solution of the differential equation

$$
\mathrm{q}(z)+\frac{z \mathrm{q}^{\prime}(z)}{\mathrm{h}(z)+\frac{\gamma+\beta-\mu-\lambda}{\mu+\lambda}}=\mathrm{h}(z), \mathrm{q}(0)=1 .
$$

Proof. Because

$$
\mathcal{S} \mathcal{V}_{m, \lambda}^{n, v}(\alpha, \beta, \mu)(h)=\left\{f \in A: \frac{z\left(I_{\lambda}^{n, v}(\alpha, \beta, \mu) f(z)\right)^{\prime}}{I_{\lambda}^{n, v}(\alpha, \beta, \mu) f_{m}(z)} \prec h(z), z \in \mathbb{U}\right\} .
$$

It is remaining to show that $f_{m} \in \mathcal{S} \mathcal{V}_{m, \lambda}^{n, v}(\alpha, \beta, \mu)$, since

$$
\left.f_{m}(z)=\frac{1}{2 m} \sum_{k=0}^{m-1}\left\{\omega^{-k} f\left(\omega^{k} z\right)+\omega^{k} \overline{f\left(\omega^{k} \bar{z}\right.}\right)\right\} \text {. }
$$

As

$$
\begin{aligned}
f \in \mathcal{S} \mathcal{V}_{m, \lambda}^{n, v}(\alpha, \beta, \mu) & \Rightarrow \frac{z\left(I_{\lambda}^{n, v}(\alpha, \beta, \mu) f(z)\right)^{\prime}}{I_{\lambda}^{n, v}(\alpha, \beta, \mu) f_{m}(z)} \prec h(z) \\
& \Rightarrow \frac{\left(D_{\lambda}^{n, v}(\alpha, \beta, \mu) z f^{\prime}(z)\right)}{I_{\lambda}^{n, v}(\alpha, \beta, \mu) f_{m}(z)} \prec h(z) .
\end{aligned}
$$

After replacing $z$ by $\omega^{j} z$ and $\omega^{j} \bar{z}$, we get

$\omega^{-j} \frac{\left(I_{\lambda}^{n, v}(\alpha, \beta, \mu) z f^{\prime}\left(\omega^{j} z\right)\right)}{I_{\lambda}^{n, v}(\alpha, \beta, \mu) f_{m}(z)} \prec h(z)$, and $\omega^{j} \frac{\overline{\left(I_{\lambda}^{n, v}(\alpha, \beta, \mu) z f^{\prime}\left(\omega^{j} \bar{z}\right)\right)}}{I_{\lambda}^{n, v}(\alpha, \beta, \mu) f_{m}(z)} \prec h(z)$

because

$$
\begin{gathered}
\overline{h(\bar{z})}=h(z), I_{\lambda}^{n, v}(\alpha, \beta, \mu) f_{m}(\bar{z})=\overline{I_{\lambda}^{n, v}(\alpha, \beta, \mu) f_{m}(z)} \text {, and } \\
I_{\lambda}^{n, v}(\alpha, \beta, \mu) f_{m}\left(\omega^{j} z\right)=\omega^{j} I_{\lambda}^{n, v}(\alpha, \beta, \mu) f_{m}(z) .
\end{gathered}
$$

Using (19) we have

$$
\frac{1}{2 m} \sum_{k=0}^{k-1} \omega^{-j} \frac{\left(I_{\lambda}^{n, v}(\alpha, \beta, \mu) z f^{\prime}\left(\omega^{j} z\right)\right)}{I_{\lambda}^{n, v}(\alpha, \beta, \mu) f_{m}(z)}+\omega^{j} \frac{\overline{\left(I_{\lambda}^{n, v}(\alpha, \beta, \mu) z f^{\prime}\left(\omega^{j} \bar{z}\right)\right)}}{I_{\lambda}^{n, v}(\alpha, \beta, \mu) f_{m}(z)} \prec h(z),
$$

implies

$$
\frac{1}{2 m} \sum_{k=0}^{k-1} \omega^{-j} \frac{z\left(I_{\lambda}^{n, v}(\alpha, \beta, \mu) f\left(\omega^{j} z\right)\right)^{\prime}}{I_{\lambda}^{n, v}(\alpha, \beta, \mu) f_{m}(z)}+\omega^{j} \frac{\overline{z\left(I_{\lambda}^{n, v}(\alpha, \beta, \mu) f\left(\omega^{j} \bar{z}\right)\right)^{\prime}}}{I_{\lambda}^{n, v}(\alpha, \beta, \mu) f_{m}(z)} \prec h(z),
$$


since we have

$$
\begin{aligned}
& z\left(\mathrm{I}_{\lambda}^{\mathrm{n}, v}(\alpha, \beta, \mu) \mathrm{f}_{\mathrm{m}}(z)\right)^{\prime} \\
& \left.\quad=\frac{1}{2 m} \sum_{k=0}^{\mathrm{m}-1}\left\{\omega^{-k} z\left(\mathrm{I}_{\lambda}^{\mathrm{n}, v}(\alpha, \beta, \mu) \mathrm{f}\left(\omega^{\mathrm{k}} z\right)\right)^{\prime}+\omega^{k} z\left(\overline{\mathrm{I}_{\lambda}^{n, v}(\alpha, \beta, \mu) \mathrm{f}\left(\omega^{k} \bar{z}\right.}\right)\right)^{\prime}\right\}
\end{aligned}
$$

and this implies

$$
\begin{aligned}
& \frac{1}{2 m} \sum_{k=0}^{k-1} \omega^{-j} \frac{z\left(I_{\lambda}^{n, v}(\alpha, \beta, \mu) f\left(\omega^{j} z\right)\right)^{\prime}}{I_{\lambda}^{n, v}(\alpha, \beta, \mu) f_{m}(z)}+\omega^{j} \frac{\overline{z\left(I_{\lambda}^{n, v}(\alpha, \beta, \mu) f\left(\omega^{j} \bar{z}\right)\right)^{\prime}}}{I_{\lambda}^{n, v}(\alpha, \beta, \mu) f_{m}(z)} \\
& \quad=\frac{z\left(I_{\lambda}^{n, v}(\alpha, \beta, \mu) f_{m}(z)\right)^{\prime}}{I_{\lambda}^{n, v}(\alpha, \beta, \mu) f_{m}(z)},
\end{aligned}
$$

therefore

$$
\frac{z\left(I_{\lambda}^{n, v}(\alpha, \beta, \mu) f_{m}(z)\right)^{\prime}}{I_{\lambda}^{n, v}(\alpha, \beta, \mu) f_{m}(z)} \prec h(z) .
$$

Hence (16) is satisfied.

From (5) we have

$$
I_{\lambda}^{n, v}(\alpha, \beta, \mu) f(z)=I_{\lambda}^{\alpha}\left(I_{\lambda}^{n-1, v}(\alpha, \beta, \mu) f(z)\right),
$$

implies

$$
\begin{aligned}
I_{\lambda}^{n, v}(\alpha, \beta, \mu) f_{m}(z)= & \left(\frac{v-\mu+\beta-\lambda}{v+\beta}\right)\left(I_{\lambda}^{n-1, v}(\alpha, \beta, \mu)\left(\Omega^{\alpha} f_{m}(z)\right)\right. \\
& +\left(\frac{\mu+\lambda}{v+\beta}\right) z\left(I_{\lambda}^{n-1, v}(\alpha, \beta, \mu)\left(\Omega^{\alpha} f_{m}(z)\right)^{\prime},\right.
\end{aligned}
$$

implies

$$
\left(I_{\lambda}^{n-1, v}(\alpha, \beta, \mu)\left(\Omega^{\alpha} f_{m}(z)\right)=\frac{v+\beta}{(\mu+\lambda) z^{\left(\frac{\mu+\lambda}{v+\beta}\right)-1}} \int_{0}^{z} t^{\left(\frac{\mu+\lambda}{v+\beta}\right)-1} I_{\lambda}^{n, v}(\alpha, \beta, \mu) f_{m}(t) d t .\right.
$$

Applying Lemma 3, we have

$$
f(z)=I_{\lambda}^{n, v}(\alpha, \beta, \mu) f_{m}(z), F(z)=\left(I_{\lambda}^{n-1, v}(\alpha, \beta, \mu)\left(\Omega^{\alpha} f_{m}(z)\right),\right.
$$

and

$$
\frac{z f^{\prime}(z)}{f(z)}=\frac{z\left(I_{\lambda}^{n, v}(\alpha, \beta, \mu) f_{m}(z)\right)^{\prime}}{I_{\lambda}^{n, v}(\alpha, \beta, \mu) f_{m}(z)} \prec h(z)(\text { proved })
$$


with

$$
\mathfrak{R}\left(\mathrm{h}(z)+\frac{\nu+\beta-\mu-\lambda}{\mu+\lambda}\right)>0 .
$$

Therefore

$$
\frac{z\left(I _ { \lambda } ^ { n - 1 , v } \left(\alpha, \beta, \mu\left(\left(\Omega^{\alpha} f_{m}(z)\right)\right)^{\prime}\right.\right.}{I_{\lambda}^{n-1, v}(\alpha, \beta, \mu)\left(\Omega^{\alpha} f_{m}(z)\right)} \prec q(z),
$$

and $\mathrm{q}$ satisfied the equation

$$
\mathrm{q}(z)+\frac{z \mathrm{q}^{\prime}(z)}{\mathrm{h}(z)+\frac{v+\beta-\mu-\lambda}{\mu+\lambda}}=\mathrm{h}(z) .
$$

Hence proved.

Corollary 1 Let $\mathfrak{R}(\mathrm{h}(z))>0$, if $\mathrm{f} \in \mathcal{S} \mathcal{V}_{\mathrm{m}, \lambda}^{\mathrm{n}, v}(\alpha, \beta, \mu)(\mathrm{h})$ then $\mathrm{I}_{\lambda}^{\mathrm{n}, v}(\alpha, \beta, \mu) \mathrm{f}_{\mathrm{m}} \in$ $S$ and hence $I_{\lambda}^{n, v}(\alpha, \beta, \mu) f$ is close to convex function.

Theorem 2 Let $\mathfrak{R}(\mathrm{h}(z)>0$ and $\overline{\mathrm{h}(\bar{z})}=\mathrm{h}(z)$ then the following inclusions hold

$$
\mathcal{S} \mathcal{V}_{m, \lambda}^{\mathrm{n}+1, v}(\alpha, \beta, \mu)(h) \subseteq \mathcal{S} \mathcal{V}_{\mathrm{m}, \lambda}^{\mathrm{n}, \mathrm{v}}(\alpha, \beta, \mu)(h) \subseteq \mathcal{S} \mathcal{V}_{\mathrm{m}, \lambda}^{\mathrm{n}-1, v}(\alpha, \beta, \mu)(h)
$$

Proof. Let $f \in \mathcal{S} \mathcal{V}_{m, \lambda}^{n+1, v}(\alpha, \beta, \mu)(h)$. To prove $\mathcal{S} \mathcal{V}_{m, \lambda}^{n+1, v}(\alpha, \beta, \mu)(h) \subseteq \mathcal{S} \mathcal{V}_{m, \lambda}^{n, v}$ $(\alpha, \beta, \mu)(h)$, it is enough to show that $f \in \mathcal{S} \mathcal{V}_{m, \lambda}^{n, v}(\alpha, \beta, \mu)(h)$.

Applying Theorem 1 , if $f \in \mathcal{S} \mathcal{V}_{m, \lambda}^{n+1, v}(\alpha, \beta, \mu)(h)$ then

$$
\frac{z\left(I_{\lambda}^{n+1, v}(\alpha, \beta, \mu) f_{m}(z)\right)^{\prime}}{I_{\lambda}^{n+1, v}(\alpha, \beta, \mu) f_{m}(z)} \prec h(z), z \in \mathbb{U}
$$

if Moreover, if $\mathfrak{R}\left(h(z)+\frac{\gamma+\beta-\mu-\lambda}{\mu+\lambda}\right)>0$ in $\mathbb{U}$ then

$$
\frac{z\left(I _ { \lambda } ^ { n , v } \left(\alpha, \beta, \mu\left(\left(\Omega^{\alpha} f_{m}(z)\right)\right)^{\prime}\right.\right.}{I_{\lambda}^{n, v}(\alpha, \beta, \mu)\left(\Omega^{\alpha} f_{m}(z)\right)} \prec q(z) \prec h(z), z \in \mathbb{U},
$$

and $\mathfrak{R}\left(\mathrm{q}(z)+\frac{\gamma+\beta-\mu-\lambda}{\mu+\lambda}\right)>0$ in $\mathbb{U}$. Let

$$
p(z)=\frac{z\left(I _ { \lambda } ^ { n , v } \left(\alpha, \beta, \mu\left(\left(\Omega^{\alpha} f(z)\right)\right)^{\prime}\right.\right.}{I_{\lambda}^{n, v}(\alpha, \beta, \mu)\left(\Omega^{\alpha} f_{m}(z)\right)} .
$$


Then $p$ is analytic in $\mathrm{U}$ and satisfies

$$
p(z)+\frac{z p^{\prime}(z)}{q(z)+\frac{v+\beta-\mu-\lambda}{\mu+\lambda}} \prec h(z),
$$

by using Lemma $2 p(z) \prec h(z)$, implies $\Omega^{\alpha} f(z) \in \mathcal{S} \mathcal{V}_{m, \lambda}^{n, v}(\alpha, \beta, \mu)(h)$, applying Theorem 4 implies $f(z) \in \mathcal{S V}_{\mathrm{m}, \lambda}^{\mathrm{n}, \boldsymbol{V}}(\alpha, \beta, \mu)(\mathrm{h})$. Hence

$$
\mathcal{S} \mathcal{V}_{m, \lambda}^{\mathrm{n}+1, v}(\alpha, \beta, \mu)(h) \subseteq \mathcal{S} \mathcal{V}_{m, \lambda}^{\mathrm{n}, v}(\alpha, \beta, \mu)(h)
$$

Similarly we can show that $\mathcal{S V}_{m, \lambda}^{\mathrm{n}, \boldsymbol{v}}(\alpha, \beta, \mu)(\mathrm{h}) \subseteq \mathcal{S V}_{\mathrm{m}, \lambda}^{\mathrm{n}-1, v}(\alpha, \beta, \mu)(\mathrm{h})$, and $\mathcal{S} \mathcal{V}_{\mathrm{m}, \lambda}^{\mathrm{n}-1, v}(\alpha, \beta, \mu)(\mathrm{h}) \subseteq \mathcal{S V}_{\mathrm{m}, \lambda}^{\mathrm{n}-2, v}(\alpha, \beta, \mu)(\mathrm{h})$ and so on, therefore

$$
\mathcal{S} \mathcal{V}_{m, \lambda}^{\mathrm{n}+1, v}(\alpha, \beta, \mu)(h) \subseteq \mathcal{S} \mathcal{V}_{m, \lambda}^{n, v}(\alpha, \beta, \mu)(h) \subseteq \mathcal{S} \mathcal{V}_{m, \lambda}^{n-1, v}(\alpha, \beta, \mu)(h) . s,
$$

which generalized the Al-Amiri et al. [16] results.

Corollary 2 Taking $\mathrm{h}(z)=\frac{1+z}{1-z}$, in Theorem 2, then

$\mathcal{S} \mathcal{V}_{m, \lambda}^{n+1, v}(\alpha, \beta, \mu)\left(\frac{1+z}{1-z}\right) \subseteq \mathcal{S} \mathcal{V}_{\mathfrak{m}, \lambda}^{n, v}(\alpha, \beta, \mu)\left(\frac{1+z}{1-z}\right) \subseteq \mathcal{S V}_{m, \lambda}^{n-1, v}(\alpha, \beta, \mu)\left(\frac{1+z}{1-z}\right)$

Implies that $\mathcal{S} \mathcal{V}_{m, \lambda}^{n, v}(\alpha, \beta, \mu)\left(\frac{1+z}{1-z}\right)$ are starlike functions with respect to symmetric conjugate points.

Theorem 3 If $f \in \mathcal{S V}_{m, \lambda}^{n, v}(\alpha, \beta, \mu)(h)$ then $f * g \in \mathcal{S V}_{m, \lambda}^{n, v}(\alpha, \beta, \mu)(h)$ where $\mathfrak{R}(\mathrm{h}(z))>0$ and $\mathrm{g}$ is a convex function with real coefficients in $\mathbb{U}$.

Proof. Since $f \in \mathcal{S} \mathcal{V}_{m, \lambda}^{n, v}(\alpha, \beta, \mu)(h)$, applying Theorem 1 , we get $I_{\lambda}^{n, v}(\alpha, \beta, \mu)$ $f_{m}(z) \in S$. Using the convolution properties we have

$$
\begin{aligned}
& \frac{z\left(I_{\lambda}^{\mathrm{n}, v}(\alpha, \beta, \mu)(f * g)(z)\right)^{\prime}}{I_{\lambda}^{\mathrm{n}, v}(\alpha, \beta, \mu)\left(f_{\mathrm{m}} * g\right)(z)}=\frac{z\left(I_{\lambda}^{\mathrm{n}, v}(\alpha, \beta, \mu)(f(z) * g(z))\right)^{\prime}}{I_{\lambda}^{\mathrm{n}, v}(\alpha, \beta, \mu)\left(f_{m}(z) * g(z)\right)} \\
& =\frac{g(z) *\left(z\left(I_{\lambda}^{\mathrm{n}, v}(\alpha, \beta, \mu) f(z)\right)^{\prime}\right.}{g(z) * I_{\lambda}^{\mathrm{n}, v}(\alpha, \beta, \mu) f_{m}(z)} \\
& =\frac{g(z) *\left(z\left(I_{\lambda}^{\mathrm{n}, v}(\alpha, \beta, \mu) f(z)\right)^{\prime} / I_{\lambda}^{\mathrm{n}, v}(\alpha, \beta, \mu) f_{m}(z)\right) I_{\lambda}^{\mathrm{n}, v}(\alpha, \beta, \mu) f_{m}(z)}{g(z) * I_{\lambda}^{n, v}(\alpha, \beta, \mu) f_{m}(z)} \\
& =\frac{g(z) * I_{\lambda}^{n, v}(\alpha, \beta, \mu) f_{m}(z)\left(z\left(I_{\lambda}^{n, v}(\alpha, \beta, \mu) f(z)\right)^{\prime} / I_{\lambda}^{n, v}(\alpha, \beta, \mu) f_{m}(z)\right)}{g(z) * I_{\lambda}^{n, v}(\alpha, \beta, \mu) f_{m}(z)} .
\end{aligned}
$$


Implies that

$$
\frac{z\left(I_{\lambda}^{n, v}(\alpha, \beta, \mu)(f * g)(z)\right)^{\prime}}{I_{\lambda}^{n, v}(\alpha, \beta, \mu)\left(f_{m} * g\right)(z)} \in \overline{c o}\left(\frac{z\left(I_{\lambda}^{n, v}(\alpha, \beta, \mu) f\right)^{\prime}}{I_{\lambda}^{n, v}(\alpha, \beta, \mu) f_{m}}(U)\right) \subseteq h(U), \quad z \in U .
$$

Hence

$$
f * g \in \mathcal{S} \mathcal{V}_{m, \lambda}^{n, v}(\alpha, \beta, \mu)(h)
$$

Theorem 4 If $\Omega^{\alpha} f(z) \in \mathcal{S} \mathcal{V}_{m, \lambda}^{n, v}(\alpha, \beta, \mu)(h)$ then $f(z) \in \mathcal{S} \mathcal{V}_{m, \lambda}^{n, v}(\alpha, \beta, \mu)(h)$, where $\mathfrak{R}(\mathrm{h}(z))>0, \overline{\mathrm{h}(\bar{z})}=\mathrm{h}(z)$.

Proof. Let $\Omega^{\alpha} f(z) \in \mathcal{S} \mathcal{V}_{m, \lambda}^{\mathfrak{n}, v}(\alpha, \beta, \mu)(h)$, applying Theorem 1, implies

$$
\frac{z\left(I_{\lambda}^{n, v}(\alpha, \beta, \mu) \Omega^{\alpha} f_{m}(z)\right)^{\prime}}{I_{\lambda}^{n, v}(\alpha, \beta, \mu) \Omega^{\alpha} f_{m}(z)} \prec h(z),
$$

or

$$
\left(I_{\lambda}^{n, v}(\alpha, \beta, \mu) \Omega^{\alpha} f_{m}(z) \in S .\right.
$$

Because

$$
\Omega^{\alpha} f(z)=\varphi(2,2-\alpha ; z) * f(z)
$$

and

$$
\mathrm{I}_{\lambda}^{\mathrm{n}, v}(\alpha, \beta, \mu) \mathrm{f}(z)=\underbrace{\left[\varphi(2,2-\alpha ; z) * \mathrm{~g}_{\beta, \lambda}^{\mu, \nu}(z) . s \varphi(2,2-\alpha ; z) * \mathrm{~g}_{\beta, \lambda}^{\mu, \nu}(z)\right]} * \mathrm{f}(z),
$$

therefore we can write that

$$
\begin{aligned}
I_{\lambda}^{n, v}(\alpha, \beta, \mu) f_{m}(z) & =\varphi(2-\alpha, 2 ; z) * D_{\lambda}^{n, v}(\alpha, \beta, \mu)\left(\Omega^{\alpha} f_{m}(z)\right) \\
z\left(I_{\lambda}^{n, v}(\alpha, \beta, \mu) f(z)\right)^{\prime} & =\varphi(2-\alpha, 2 ; z) * z\left(I_{\lambda}^{n, v}(\alpha, \beta, \mu)\left(\Omega^{\alpha} f(z)\right)^{\prime},\right.
\end{aligned}
$$

where $\varphi(2-\alpha, 2 ; z) \in \mathrm{K}$.

Using (20) we have

$$
\begin{aligned}
& \frac{z\left(I_{\lambda}^{n, v}(\alpha, \beta, \mu) f(z)\right)^{\prime}}{I_{\lambda}^{n, v}(\alpha, \beta, \mu) f_{m}(z)}=\frac{\varphi(2-\alpha, 2 ; z) * z\left(I_{\lambda}^{n, v}(\alpha, \beta, \mu)\left(\Omega^{\alpha} f(z)\right)\right)^{\prime}}{\varphi(2-\alpha, 2 ; z) * I_{\lambda}^{n, v}(\alpha, \beta, \mu)\left(\Omega^{\alpha} f_{m}(z)\right)}, \\
& \frac{z\left(I_{\lambda}^{n, v}(\alpha, \beta, \mu) f(z)\right)^{\prime}}{I_{\lambda}^{n, v}(\alpha, \beta, \mu) f_{m}(z)} \\
& \quad=\frac{\varphi(2-\alpha, 2 ; z) * I_{\lambda}^{n, v}(\alpha, \beta, \mu)\left(\Omega^{\alpha} f_{m}(z)\right) \frac{\left(z\left(I_{\lambda}^{n, v}(\alpha, \beta, \mu)\left(\Omega^{\alpha} f(z)\right)\right)^{\prime}\right.}{I_{\lambda}^{n, v}(\alpha, \beta, \mu)\left(\Omega^{\alpha} f_{m}(z)\right)}}{\varphi(2-\alpha, 2 ; z) * I_{\lambda}^{n, v}(\alpha, \beta, \mu)\left(\Omega^{\alpha} f_{m}(z)\right)}, \\
& \frac{z\left(I_{\lambda}^{n, v}(\alpha, \beta, \mu) f(z)\right)^{\prime}}{I_{\lambda}^{n, v}(\alpha, \beta, \mu) f_{m}(z)} \in \overline{c o}\left(\frac{z\left(I_{\lambda}^{n, v}(\alpha, \beta, \mu) \Omega^{\alpha} f\right)^{\prime}}{I_{\lambda}^{n, v}(\alpha, \beta, \mu) \Omega^{\alpha} f_{m}}(\mathbb{U})\right) \subseteq h(\mathbb{U}), \quad z \in \mathbb{U} .
\end{aligned}
$$


Hence

$$
f(z) \in \mathcal{S} \mathcal{V}_{m, \lambda}^{n, v}(\alpha, \beta, \mu)(h)
$$

Theorem 5 Let $0 \leq \alpha_{1}<\alpha<1$, and $\operatorname{Re}(\mathrm{h}(z))>\frac{1}{2}$, then the following inclusions hold

$$
\mathcal{S} \mathcal{V}_{m, \lambda}^{n, \nu}(\alpha, \beta, \mu)(h) \subseteq \mathcal{S} \mathcal{V}_{m, \lambda}^{\mathrm{n}, \mathcal{V}}\left(\alpha_{1}, \beta, \mu\right)(h)
$$

Proof. Let $f(z) \in \mathcal{S} \mathcal{V}_{m, \lambda}^{\mathfrak{n}, \mathcal{v}}(\alpha, \beta, \mu)(h)$, from (7) we have

$$
\mathrm{I}_{\lambda}^{\mathrm{n}, \nu}(\alpha, \beta, \mu) \mathrm{f}(z)=\underbrace{\left[\varphi(2,2-\alpha ; z) * g_{\beta, \lambda}^{\mu, v}(z) \cdot s \varphi(2,2-\alpha ; z) * g_{\beta, \lambda}^{\mu, \nu}(z)\right]} * \mathrm{f}(z),
$$

implies

$\mathrm{I}_{\lambda}^{\mathrm{n}, v}\left(\alpha_{1}, \beta, \mu\right) f(z)=\underbrace{\left[\varphi\left(2,2-\alpha_{1} ; z\right) * g_{\beta, \lambda}^{\mu, v}(z) \cdot s \varphi\left(2,2-\alpha_{1} ; z\right) * g_{\beta, \lambda}^{\mu, v}(z)\right]} * f(z)$,

$$
I_{\lambda}^{\mathrm{n}, v}\left(\alpha_{1}, \beta, \mu\right) f(z)=\underbrace{\left[\varphi\left(2-\alpha, 2-\alpha_{1} ; z\right) * . s * \varphi\left(2-\alpha, 2-\alpha_{1} ; z\right)\right]}_{* I_{\lambda}^{\mathrm{n}, v}(\alpha, \beta, \mu) f(z),}
$$

implies that

$$
z\left(\mathrm{I}_{\lambda}^{\mathrm{n}, v}\left(\alpha_{1}, \beta, \mu\right) f(z)\right)^{\prime}=\underbrace{\left[\varphi\left(2-\alpha, 2-\alpha_{1} ; z\right) * . s * \varphi\left(2-\alpha, 2-\alpha_{1} ; z\right)\right]}_{* z\left(\mathrm{I}_{\lambda}^{\mathrm{n}, v}(\alpha, \beta, \mu) \mathrm{f}(z)\right)^{\prime},}
$$

applying same technique, we have

$$
I_{\lambda}^{n, v}\left(\alpha_{1}, \beta, \mu\right) f_{m}(z)=\underbrace{\left[\varphi\left(2-\alpha, 2-\alpha_{1} ; z\right) * . s * \varphi\left(2-\alpha, 2-\alpha_{1} ; z\right)\right]}_{* I_{\lambda}^{n, v}(\alpha, \beta, \mu) f_{m}(z),}
$$

since Y. Ling and S. Ding [24] already proved that $\varphi\left(2-\alpha, 2-\alpha_{1} ; z\right) \in S(1 / 2)$, therefore by Lemma 1 we get $\left[\varphi\left(2-\alpha, 2-\alpha_{1} ; z\right) * . S * \varphi\left(2-\alpha, 2-\alpha_{1} ; z\right)\right] \in S(1 / 2)$.

From last two equations we get

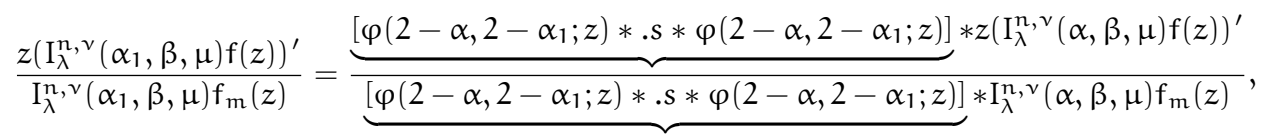


after simplification and using Lemma 5 we deduce that

$$
\frac{z\left(I_{\lambda}^{n, v}\left(\alpha_{1}, \beta, \mu\right) f(z)\right)^{\prime}}{I_{\lambda}^{n, v}\left(\alpha_{1}, \beta, \mu\right) f_{m}(z)} \in \overline{c o}\left(\frac{z\left(I_{\lambda}^{n, v}(\alpha, \beta, \mu) f\right)^{\prime}}{I_{\lambda}^{n, v}(\alpha, \beta, \mu) f_{m}}(\mathbb{U})\right) \subseteq h(\mathbb{U}), \quad z \in \mathbb{U}
$$

therefore

$$
f \in \mathcal{S} \mathcal{V}_{m, \lambda}^{n, v}\left(\alpha_{1}, \beta, \mu\right)(h)
$$

Hence proved.

Remark 2 1. When $v=1, \mu=\beta=0, \mathcal{S V}_{\mathrm{m}, \lambda}^{\mathrm{n}, 1}(\alpha, 0,0)(\mathrm{h})=\mathcal{S} \mathcal{V}_{\mathrm{m}, \lambda}^{\mathrm{n}}(\alpha)(\mathrm{h})$, the classes of functions related to starlike functions with respect to symmetric conjugate points, defined and studied by M. K. Al-Oboudi [25].

2. For $\mathrm{n}=\mathrm{m}=\mathrm{v}=1, \mu=\beta=0$, and $\mathrm{h}(z)=\frac{1+z}{1-z}, \mathcal{S} \mathcal{V}_{1, \lambda}^{1,1}(\alpha, 0,0)\left(\frac{1+z}{1-z}\right)=$ $\mathcal{S} \mathcal{V}^{\lambda, \alpha}\left(\frac{1+z}{1-z}\right)$, the class of $\lambda$-starlike functions with respect to conjugate points, defined and studied by Radha [23].

3. For $\mathrm{n}=\mathrm{m}=\mathrm{v}=\lambda=1, \alpha=\mu=\beta=0$, and $\mathrm{h}(z)=\frac{1+z}{1-z}, \mathcal{S V}_{1,1}^{1,1}(0,0,0)$ $\left(\frac{1+z}{1-z}\right)=\mathcal{S} \mathcal{V}\left(\frac{1+z}{1-z}\right)$, the class of starlike functions with respect to conjugate points, defined and studied by El-Ashwah and Thomas [5].

4. For $\mathrm{n}=\mathrm{v}=1, \alpha=\mu=\beta=0$, and $\mathrm{h}(z)=\frac{1+z}{1-z}, \mathcal{S V}_{\mathrm{m}, \lambda}^{1,1}(0,0,0)\left(\frac{1+z}{1-z}\right)=$ $\mathcal{S} \mathcal{V}^{\mathrm{m}, \lambda}\left(\frac{1+z}{1-z}\right)$, the class of $\lambda$-starlike functions with respect to $2 \mathrm{~m}$-symmetric conjugate points, defined and studied by Al-Amiri et al. $[15,16]$.

5. For $\mathrm{m}=\boldsymbol{v}=1, \mathrm{n}=\mu=\beta=0, \mathcal{S V}_{1, \lambda}^{0,1}(\alpha, 0,0)(\mathrm{h})=\mathcal{S} \mathcal{V}(\alpha)(\mathrm{h})$, the class of functions related to starlike functions with respect to conjugate points, defined and studied by Ravichandran [31].

6. For $\mathrm{m}=\mathrm{n}=\mathrm{v}=1, \alpha=\mu=\beta=0$ and $\mathrm{h}(z)=\frac{1+z}{1-z}, \mathcal{S} \mathcal{V}_{1, \lambda}^{1,1}(0,0,0)\left(\frac{1+z}{1-z}\right)=$ $\mathcal{S} \mathcal{V}^{\lambda}\left(\frac{1+z}{1-z}\right)$, the class of $\lambda$-starlike functions with respect to conjugate points, defined and studied by Radha [23].

7. For $\lambda=n=v=1, \alpha=\mu=\beta=0$ and $h(z)=\frac{1+z}{1-z}, \mathcal{S} \mathcal{V}_{m, 1}^{1,1}(0,0,0)(h)=$ $\mathcal{S} \mathcal{V}^{\mathrm{m}}\left(\frac{1+z}{1-z}\right)$, the class of starlike functions with respect to $2 \mathrm{~m}$-symmetric conjugate points, defined by Al-Amiri et al. [16].

8. For $\boldsymbol{v}=1, \mu=\beta=0, \mathcal{K} \mathcal{V}_{m, \lambda}^{\mathrm{n}, 1}(\alpha, 0,0)(\mathrm{h})=\mathcal{K} \mathcal{V}_{\lambda}^{\mathrm{m}, \mathrm{n}}(\alpha)(\mathrm{h})$, the classes of functions related to starlike functions with respect to symmetric conjugate points, defined and studied by M.K. Al-Oboudi [25]. 
9. For $\mathrm{n}=\mathrm{v}=1, \alpha=\mu=\beta=0$ and $\mathrm{h}(z)=\frac{1+z}{1-z}, \mathcal{K} \mathcal{V}_{\mathrm{m}, \lambda}^{1,1}(0,0,0)\left(\frac{1+z}{1-z}\right)=$ $\mathcal{K} \mathcal{V}_{\lambda}^{\mathrm{m}}\left(\frac{1+z}{1-z}\right)$, the class of $\lambda$-close to convex functions with respect to symmetric conjugate points, defined and studied by Al-Amiri et al. [16].

10. For $\mathrm{m}=\mathrm{n}=v=1, \alpha=\mu=\beta=0$ and $\mathrm{h}(z)=\frac{1+z}{1-z}, \mathcal{K} \mathcal{V}_{1, \lambda}^{1,1}(0,0,0)\left(\frac{1+z}{1-z}\right)=$ $\mathcal{K} \mathcal{V}^{\lambda}\left(\frac{1+z}{1-z}\right)$, the class of $\lambda$-close to convex functions with respect to symmetric conjugate points, defined and studied by Radha [23].

\section{Acknowledgments}

The work presented here was fully supported by UKM-AP-2013-09.

\section{References}

[1] K. Sakaguchi, On certain univalent mappings, J. Math. Soc. Japan, 11 (1959), 72-75.

[2] T. N. Shanmugam, C. Ramachandran, V. Ravichandran, Fekete-Szego problem for subclasses of starlike functions with respect to symmetric points, Bull. Korean Math. Soc., 43 (2006), 589-598.

[3] R. Chand, P. Singh, On certain schlicht mappings, J. Pure Appl. Math., 10(9) (1979), 1167-1174.

[4] R. N. Das, P. Singh, On subclasses of schlicht mapping, Indian J. Pure Appl. Math., 8 (8) (1977), 864-872.

[5] M. El-Ashwah, D. K. Thomas, Some subclasses of close-to-convex functions, J. Ramanujan Math. Soc., 2 (1) (1987), 85-100.

[6] S. Owa, On the distortion theorems, I. Kyungpook Math J., 18 (1) (1978), $53-59$.

[7] S. Owa, H. M. Srivastava, Univalent and starlike generalized hypergeometric functions, Canad. J. Math., 39 (5) (1987), 1057-1077.

[8] M. K. Aouf, R. M. El-Ashwah, S. M. El-Deeb, Some inequalities for certain p-valent functions involving extended multiplier transformations, Proc. Pak. Acad. Sci., 46 (4) (2009), 217-221.

[9] F. M. Al-Oboudi, On univalent functions defined by a generalized Salagean operator, Int. J. Math. Math. Sci., (2004), 1419-1436. 
[10] G. S. Salagean, Subclasses of univalent functions, Lecture Notes in Mathematics 1013, Springer-Verlag, 1983.

[11] B. A. Uralegaddi, C. Somanatha, Certain classes of univalent functions, In: Current Topics in Analytic Function Theory, Eds. Srivastava, H. M. and Owa, S., World Scientific Publishing Company, Singapore, 1992.

[12] N. E. Cho, H. M. Srivastava, Argument estimates of certain analytic functions defined by a class of multiplier transformations, Math. Comput. Modeling, 37 (2003), 39-49.

[13] N. E. Cho, T. H. Kim, Multiplier transformations and strongly close-toconvex functions, Bull. Korean Math. Soc., 40 (2003), 399-410.

[14] F. M. Al-Oboudi, K.A . Al-Amoudi, On classes of analytic functions related to conic domains, J. Math. Anal. Appl., 399 (2008), 655-667.

[15] H. S. Al-Amiri, D. Coman, P. T. Mocanu, Some properties of starlike functions with respect to symmetric- conjugate points, Int. J. Math. Math. Sci., 18 (3) (1995), 467-474.

[16] H. S. Al-Amiri, B. Green, D. Coman, P. T. Mocanu, Starlike and close-toconvex functions with respect to symmetric-conjugate points, Glas. Math. III. Ser., 30 (2) (1995), 209-219.

[17] P. T. Mocanu, On starlike functions with respect to symmetric points, Bull. Math. Soc. Sci. Math. Roum. Nouv. Sr., 28 (1) (1984), 47-50.

[18] P. T. Mocanu, Certain classes of starlike functions with respect to symmetric points, Mathematica (Cluj), 32 (55) (1990), 153-157.

[19] St. Ruscheweyh, Sheil-Small, Hadamard products of schlicht functions and the Polya-Schoenberg conjecture, Comment. Math. Helv., 48 (1973), 119-135.

[20] S. S Miller, P. T. Mocanu, General second order inequalities in the complex plane, Babes-Bolyai Univ. Fac. of Math. Research Seminars, Seminar on Geometric Function Theory, 4 (1982), 96-114.

[21] S. S Miller, P. T. Mocanu, Differnetial Subordination Theory and Applications, Marcel Dekker Inc. New York, 2000.

[22] St. Ruscheweyh, Convolutions in Geometric Function Theory, Sem. Math. Su., vol. 83. Presses Univ. de Montreal, 1982. 
[23] S. Radha, On $\alpha$-starlike and $\alpha$-close-to-convex functions with respect to conjugate points, Bull. Inst. Math. Acad. Sinica, 18 (1990), 41-47.

[24] Y. Ling, S. Ding, A class of analytic functions defined by fractional derivative, J. Math. Anal. Appl., 186 (1994), 504-513.

[25] F. M. Al-Oboudi, On classes of functions related to starlike functions with respect to symmetric conjugate points defined by a fractional differential operator, Complex Anal. Oper. Theory, DOI 10.1007/s11785-010-0069-2.

[26] A. Catas, On certain classes of p-valent functions defined by multiplier transformations, in: S. Owa, Y. Polatoglu (Eds.), Proceedings of the International Symposium on Geometric Function Theory and Applications:, GFTA 2007 Proceedings, Istanbul, Turkey, 20-24 August 2007, vol. 91, TC Istanbul Kultur University Publications, TC Istanbul Kultur University, Istanbul, Turkey, 2008.

[27] S. S. Kumar, H. C. Taneja, V. Ravichandran, Classes multivalent functions defined by Dziok-Srivastava linear operaor and multiplier transformations, Kyungpook Math. J., 46 (2006), 97-109.

[28] H. M. Srivastava, K. Suchithra, B. Adolf Stephen, S. Sivasubramanian, Inclusion and neighborhood properties of certain subclasses of multivalent functions of complex order, J. Ineq. Pure Appl. Math., 5 (7) (2006), 1-8.

[29] M. Darus, I. Faisal, A different approach to normalized analytic aunctions through meromorphic functions defined by extended multiplier transformations operator, Int. J. App. Math. Stat., 23 (11) (2011), 112-121.

[30] M. Darus, I. Faisal, Characrerization properties for a class of analytic aunctions defined by generalized Cho and Srivastava operator, In Proc. 2nd Inter. Conf. Math. Sci., Kuala Lumpur, Malaysia, (2010), 1106-1113.

[31] V. Ravichandran, Starlike and convex functions with respect to conjugate points, Acta Mathematica Academiae Paedagogicae Nyiregyaziensis 20 (2004), 31-37. 